\title{
Effect of Multimodal Intervention Care on Cachexia in Patients with Advanced Cancer Compared To Conventional Management (MIRACLE): An Open- Label, Parallel, Randomized, Phase 2 Trial
}

Chi Hoon Maeng ( $\nabla$ mchihoon@khu.ac.kr)

Kyung Hee University Hospital https://orcid.org/0000-0002-4450-3350

Bo-Hyung Kim

Kyung Hee University Hospital

Jinmann Chon

Kyung Hee University Hospital

\section{Won Sub Kang}

Kyung Hee University Hospital

Kyounglan Kang

Kyung Hee University Hospital

Mihye Woo

Kyung Hee University Hospital

II Ki Hong

Kyung Hee University Hospital

Junhee Lee

Kyung Hee University College of Korean Medicine

Kil Yeon Lee

Kyung Hee University Hospital

\section{Research Article}

Keywords: cachexia, cancer, chemotherapy, ibuprofen, exercise, omega-3-fatty acid, complementary and alternative medicine

Posted Date: December 8th, 2021

DOI: https://doi.org/10.21203/rs.3.rs-882546/v1

License: (c) (i) This work is licensed under a Creative Commons Attribution 4.0 International License. Read Full License 
Version of Record: A version of this preprint was published at Trials on April 11th, 2022. See the published version at https://doi.org/10.1186/s13063-022-06221-z. 


\section{Abstract}

Background: the context and purpose of the study

Cancer cachexia (CC) is a multifactorial process characterized by progressive weight loss, muscle mass, and fat tissue wasting, which adversely affects the quality of life and survival of patients with advanced stages of cancer. CC has a complex and multifactorial pathophysiology, and there is no established standard treatment. Therefore, it is often irreversible and a single treatment modality is unlikely to suppress its progression. We are conducting a randomized trial to investigate the efficacy and safety of a multimodal intervention compared to the best supportive care for patients who received palliative chemotherapy.

Methods: how the study will be performed

Patients with lung or gastrointestinal cancers undergoing palliative chemotherapy are eligible. Patients are randomized into a multimodal intervention care (MIC) arm versus a conventional palliative care (CPC) arm. MIC includes ibuprofen, omega-3-fatty acid, oral nutritional supplement, weekly physical, psychiatric assessment, nutritional counseling, and complementary and alternative medicine. CPC includes basic nutritional counseling and megestrol acetate as needed (i.e., anorexia $\geq$ grade 2). All interventions are performed for 12 weeks per subject. The co-primary outcomes are median change $(\mathrm{kg})$ in total lean body mass and handgrip strength $(\mathrm{kg})$ from the baseline. A total of 112 patients will be assigned to the two arms (56 in each group).

Discussion: a brief summary and potential implications

The purpose of this study is to evaluate the effect of MIC in preventing or alleviating $\mathrm{CC}$ in patients who underwent palliative chemotherapy. As there is no established single treatment for $\mathrm{CC}$, it is expected that the results of this clinical trial will provide new insights to significantly improve the quality of life of patients with cancer. Considering the complex mechanisms of cachexia, the effect of MIC rather than a single specific drug is more promising. In this study, we did not overly restrict the type of cancer or chemotherapy. Therefore, we attempted to measure the effects of complex interventions while preserving clinical situations. Thus, it is expected that the results of this study can be applied effectively to real-world practice.

Trial registration: If your article reports the results of a health care intervention on human participants, it must be registered in an appropriate registry and the registration number and date of registration should be in stated in this section. If it was not registered prospectively (before enrollment of the first participant), you should include the words 'retrospectively registered'. See our editorial policies for more information on trial registration.

This clinical trial was registered in the Clinical Research Information Service (KCT0004967), Korean Clinical Trial Registry on April 27, 2020, and ClinicalTrial.gov (NCT 04907864) on June 1, 2021. 


\section{Introduction}

\section{Background and rationale $\{6 a\}$}

SPIRIT guidance: Description of research question and justification for undertaking the trial, including summary of relevant studies (published and unpublished) examining benefits and harms for each intervention.

Cancer cachexia (CC) is a multi-factorial process characterized by progressive weight loss, muscle mass, and fat tissue wasting, and adversely affects the quality of life and survival of patients with advanced stages of cancer (1). It is known that $30 \%-50 \%$ of patients already experience cachexia at the time of initial diagnosis, depending on the type of tumor (2). Furthermore, CC affects $50 \%-80 \%$ of patients with advanced cancer, most commonly with lung and gastrointestinal cancer, and is the main cause of death in $20 \%-30 \%$ of patients with cancer (3-5). The pathophysiology of CC can be explained by various complex mechanisms, including metabolic alterations in protein and fat, changes in hormonal activity, and tumor-driven inflammation (6). In addition, cancer treatment itself can cause or worsen cachexia due to chemotherapy-induced anorexia, poor oral intake, and decreased physical activity $(7,8)$. Therefore, it is often irreversible, and it is difficult to suppress its progression with any single treatment modality.

Megestrol acetate (MA), which can help maintain appetite and body weight in patients with advanced cancer, has not been proven to be effective in improving quality of life or lean body mass (LBM) (9). It is known that the weight gain caused by MA is mainly because of edema or an increase in adipose tissue (10). Furthermore, its use is often limited owing to various adverse events (AEs), such as Cushing syndrome, adrenal insufficiency, or thromboembolic risk (11). Anamorelin, a selective ghrelin receptor agonist, was studied for CC in patients with non-small cell lung cancer in pivotal phase 3 trials. In that study, 12 weeks of anamorelin administration had a positive effect on LBM but not on handgrip strength compared to placebo (3). However, the effect was modest, and the drug is not currently approved for CC by the United States Food and Drug Administration (FDA) and European Medicines Agency (EMA) (8). Non-steroidal anti-inflammatory drugs (NSAIDs) have been studied to reduce systemic inflammation in patients with CC. A meta-analysis revealed that NSAIDs may improve the weight of patients with CC, preserving their quality of life, although the level of evidence was weak (12). Other pharmacological interventions, such as glucocorticoids, cannabinoids, androgens, and prokinetics have been examined to improve $\mathrm{CC}$, but there is no clear evidence to date.

Of note, $\mathrm{CC}$ does not indicate a single static state, but develops as a continuous spectrum through several stages (1). If the patient is relatively early in the cancer trajectory, the symptoms and signs of cachexia can be alleviated with appropriate chemotherapy (8). However, in late-stage patients with severe muscle wasting, cachexia is often refractory and unlikely to respond to any intervention $(1,13)$. Therefore, it is necessary to develop a multimodal intervention to prevent or alleviate $\mathrm{CC}$ at an early stage in patients with advanced cancer receiving chemotherapy, even if weight loss of metabolic changes suggestive of CC has 
not yet been revealed. We are conducting an open-label, parallel, randomized phase 2 trial to investigate the efficacy and safety of a multimodal intervention including anti-inflammation, omega-3-fatty acids (O3FA), nutritional supplement with counseling, physical exercise, psychiatric intervention, as well as Bojungikki-tang, which mediates immune-modulation and reverses chronic inflammation and wasting conditions as a complementary and alternative medicine (CAM) compared to patients receiving the best supportive care.

\section{Objectives $\{7\}$}

SPIRIT guidance: Specific objectives or hypotheses.

We hypothesized that a multimodal intervention comprising anti-inflammation, 03FA, oral nutritional supplement (ONS) with counseling by a nutritionist, physical exercise, psychiatric intervention, and Bojungikki-tang, which mediates immune modulation and reverses both chronic inflammation and wasting conditions as a CAM could prevent the development of CC or improve CC in patients with advanced cancer during chemotherapy compared to in those who received the standard supportive care.

\section{Trial design $\{8\}$}

SPIRIT guidance: Description of trial design including type of trial (eg, parallel group, crossover, factorial, single group), allocation ratio, and framework (eg, superiority, equivalence, noninferiority, exploratory).

This study is an open-label, parallel, randomized phase 2 trial to determine the superiority of multi-modal intervention care (MIC) to conventional palliative care (CPC) in terms of prevention or alleviation of CC. Patients will be randomly assigned in a 1:1 ratio to either the MIC or CPC arm.

\section{Methods: Participants, Interventions And Outcomes}

\section{Study setting $\{9\}$}

SPIRIT guidance: Description of study settings (eg, community clinic, academic hospital) and list of countries where data will be collected. Reference to where list of study sites can be obtained.

This clinical trial is being conducted at the multidisciplinary departments of the Kyung Hee University Hospital, Korea. The departments of medical oncology, surgery, clinical pharmacology, psychiatry, nutrition, nuclear medicine, physical medicine, and rehabilitation are involved in patient recruitment and treatment intervention. A specialist from the Department of Sasang Constitutional Medicine of Kyung Hee University Korean Medicine Hospital is also involved in treatment intervention for CAM.

\section{Eligibility criteria $\{10\}$}

SPIRIT guidance: Inclusion and exclusion criteria for participants. If applicable, eligibility criteria for study centres and individuals who will perform the interventions (eg, surgeons, psychotherapists). 
The inclusion criteria are as follows: 1) Patients who are diagnosed with recurrent or metastatic solid cancer: gastric, colorectal, pancreatic, biliary tract, and lung. 2) Patients receiving first- or second-line palliative chemotherapy. 3) Eastern Cooperative Oncology Group performance status 0-2. 4) Patients categorized into normal, precachexia, or cachexia by pre-defined stages of CC. Precachexia is defined as weight loss $\leq 5 \%$ within the last 6 months, anorexia, and metabolic changes, such as glucose intolerance. Cachexia is defined as weight loss $>5 \%$ within the last 6 months or body mass index $<20$ and weight loss $>2 \%$ within the last 6 months (14). 5) Age $\geq 19$ years. 6) Patients who can comply with

treatment interventions in the clinical trial at the discretion of the researcher. For example, those who have no restrictions on simple daily exercise, ONS intake, and use of NSAIDs. 7) Patients with adequate organ function. Laboratory findings conducted within one week of the study enrolment should be appropriate for the treatment intervention as well as chemotherapy: total white blood cell $\geq 3,000 \mathrm{~mm}^{3}$, absolute neutrophil count $\geq 1,200 \mathrm{~mm}^{3}$, platelet $\geq 100,000 \mathrm{~mm}^{3}$, haemoglobin $\geq 8.0 \mathrm{~g} / \mathrm{dL}$, aspartate transaminase, and alanine transaminase 3 times or less of the upper limit of the reference range, total bilirubin 2 times or less of the upper limit of the reference range, serum creatinine 1.5 times or less of the upper limit of the reference range or estimated glomerular filtration rate calculated by the Cockcroft-Gault equation $>60 \mathrm{~mL} / \mathrm{min}$.

Exclusion criteria are as follows:1) Patients who have a history of cardiovascular disease with or without active treatment: congestive heart failure, poorly controlled hypertension $\geq 160 \mathrm{mmHg}$ of systolic blood pressure or $100 \mathrm{mmHg}$ of diastolic blood pressure despite antihypertensive medication, coronary heart disease. 2) Patients with bronchial asthma. 3) Patients who are unable to intake food or medication orally or have considerable difficulty in adequate oral intake: bowel obstruction, uncontrolled active inflammatory bowel disease, and short bowel syndrome. 4) Patients associated with bleeding risk or peptic ulcer disease or contraindication to taking NSAIDs. 5) Patients who have taken MA or progestin analogues within 30 days prior to the study participation. 6) Patients who are taking aspirin or NSAIDs continuously for more than 1 week at the time of the study participation. 7) Patients who are taking anticoagulants.

Prespecified medical oncologists will conduct chemotherapy for each participant and prescribe concomitant interventional drugs (NSAIDs, O3FA, and ONS) during the study period. Psychiatric interventions will be performed by a psychiatrist. The exercise program will be established by a physiatrist, and each individual participant's exercise therapy will be performed by trained, certified physical therapists. Regular nutritional counseling for participants will be provided by two nutritionists. A certified Korean medical doctor will provide herbal medication as CAM. A nuclear medicine specialist will conduct and interpret dual-energy X-ray absorptiometry (DEXA) for each participant's study outcomes, although he will not be directly involved in specific treatment intervention. Finally, surveys of patient reported outcomes, including quality of life, will be provided and conducted by medical oncologists and Korean medical doctors who will be the physicians in charge of actual medical management for the participants.

\section{Who will take informed consent? \{26a\}}


SPIRIT guidance: Who will obtain informed consent or assent from potential trial participants or authorised surrogates, and how (see Item 32).

Medical oncologists who participate as co-investigators in this study will provide informed consent. Patients treated with palliative chemotherapy are potential subjects for this study. If a patient is judged to be potentially eligible, written informed consent is obtained after giving the patient sufficient time to explain the purpose, methods, significance of the study, and the potential risks and benefits.

\section{Additional consent provisions for collection and use of participant data and biological specimens $\{26 \mathrm{~b}\}$}

SPIRIT guidance: Additional consent provisions for collection and use of participant data and biological specimens in ancillary studies, if applicable.

Not applicable

\section{Interventions}

\section{Explanation for the choice of comparators $\{6 \mathrm{~b}\}$}

SPIRIT guidance: Explanation for choice of comparators.

Participants are randomly assigned to either the "Multimodal Interventional Care" (MIC) group or "Conventional Palliative Care" (CPC) group in a 1:1 ratio.

\section{Intervention description $\{11 \mathrm{a}\}$}

SPIRIT guidance: Interventions for each group with sufficient detail to allow replication, including how and when they will be administered.

\section{Multimodal Interventional Care (MIC)}

1) Intervention with investigational product (IP)

A participant allocated to the MIC group will receive the following medication as IPs for 12 weeks while the palliative chemotherapy continues as planned: NSAID, O3FA, Bojungikki-tang, and ONS. NSAID is provided orally as ibuprofen $200 \mathrm{mg}$ three times a day. O3FA is provided orally at $1000 \mathrm{mg}$ twice a day. Bojungikki-tang is provided orally as $3.75 \mathrm{~g}$ twice a day. ONS is in the form of one sachet containing a $200 \mathrm{~mL}$ beverage designed for drinking. Each $200 \mathrm{~mL}$ ONS contains $9.8 \mathrm{~g}$ of protein, $27 \mathrm{~g}$ of carbohydrate, $6 \mathrm{~g}$ of fat, and $2 \mathrm{~g}$ of fiber. The participants will take it twice a day. All prescribed IPs should be taken daily for 12 weeks, unless a participant is intolerable to them. All IPs should be administered to the participants according to the study protocol. To check compliance with the administration of IP, the remaining IPs that the participant could not take will be retrieved at the next visit and the number of remaining medications will be checked.

2) Intervention with a psychiatrist 
Psychotherapy

Cognitive behavioral therapy for stress

\section{General psychotherapy}

Week 1: stress screening

Week 3: talking about cancer, coping with physical symptoms

Week 5: finding and changing thoughts causing stress

Week 7: learning coping skills or anger management

Week 9: coping with physical changes and potential fears
Cognitive behavioral therapy for sleep

Pharmacotherapy
Cognitive behavioral therapy for insomnia

Performed when necessary according to the researcher's clinical decision

3) Intervention through exercise by a physiatrist

Type

Warm-up exercise

Stretching

(ROM exercise)

Conditioning (Training) exercise
Intervention

5-10 min: low intensity ( $<40 \%$ of $\left.\mathrm{VO}_{2} \mathrm{Max}\right)$ or moderate intensity

10 mins

20-30 mins: aerobic exercise (ergometer or treadmill)

Resistance exercise $(60 \%-80 \%$ of $1 \mathrm{RM}, 1$ set $10-15$ repetition)

Flexibility exercise (upper \& lower extremity)

Cool-down exercise

5-10 mins: Low or moderate intensity: cardiovascular \& muscle endurance exercise

* $\mathrm{VO}_{2} \mathrm{Max}$, maximal oxygen consumption; $\mathrm{ROM}$, range of motion; $\mathrm{RM}$, repetition maximum.

4) Others

Participants will receive nutritional counseling four times (baseline, weeks 4,7 , and 13) during the study period by hospital nutrition specialists. Through detailed interviews and counseling, participants can be helped to have their daily meals at home.

\section{Conventional Palliative Care (CPC)}

Participants assigned to the CPC group will receive their planned chemotherapy with the best supportive care. No additional IP, psychiatric intervention, or exercise will be provided. However, routine nutritional counseling for all patients who are treated with chemotherapy will be provided twice (baseline and week 13) during the study period. In Korea, every patient receiving chemotherapy can be provided with two nutritional counseling sessions under the reimbursement schedule of the Korean National Health Insurance. In addition, at the clinical discretion of the researcher, MA 625 mg once a day can be 
administered if the participant shows more than grade 2 anorexia or weight loss based on the Common Terminology Criteria for Adverse Events (CTCAE) version 5.0. MA can also be provided to the participant if the subject is categorized as pre-defined precachexia or cachexia.

\section{Criteria for discontinuing or modifying allocated interventions $\{11 \mathrm{~b}\}$}

SPIRIT guidance: Criteria for discontinuing or modifying allocated interventions for a given trial participant (eg, drug dose change in response to harms, participant request, or improving/worsening disease).

Subjects may voluntarily discontinue participation in the study at any time. In addition, the investigator may discontinue the intervention or suspend the subject's participation in this study at any time according to the following criteria: 1) When a subject requests to discontinue part of the clinical trial treatment during the study period or withdraws consent to participate in this trial. 2) When the investigator decides to drop out of the study because the subject's continued participation in the study poses a risk that outweighs the potential benefit to the subject. 3) When the subject has taken a drug that is expected to affect the evaluation of the safety and efficacy of the interventions. 4) Violation of inclusion or exclusion criteria.

\section{Strategies to improve adherence to interventions $\{11 \mathrm{c}\}$}

SPIRIT guidance: Strategies to improve adherence to intervention protocols, and any procedures for monitoring adherence (eg, drug tablet return, laboratory tests).

In this study, especially when assigned to the MIC group, there is a concern that the compliance of the participants might be lowered owing to three kinds of IPs to be taken with multiple and frequent visits to the hospital for exercise intervention. To increase compliance of the participants, trained study coordinators will call the patients before each visit to remind them of the study schedule, and even when they move within the hospital (for example, to the treatment room, laboratory, or exercise therapy room), study coordinators will accompany them directly. To assess drug compliance, the remaining IPs that could not be taken as scheduled will be returned and counted at the next visit.

\section{Relevant concomitant care permitted or prohibited during the trial $\{11 \mathrm{~d}\}$}

SPIRIT guidance: Relevant concomitant care and interventions that are permitted or prohibited during the trial.

MA is not allowed to the participants allocated to the MIC group. There will be no restrictions on chemotherapy or radiation therapy required for patients during clinical trials.

\section{Provisions for post-trial care $\{30\}$}

SPIRIT guidance: Provisions, if any, for ancillary and post-trial care, and for compensation to those who suffer harm from trial participation. 
When an adverse reaction occurs during the conduct of this clinical trial, the investigators will apply utmost effort to recover the side effect by administering appropriate treatment, and to minimize the financial and social burden on the patient when hospitalization is required because of an adverse reaction directly due to trial participation. Costs for appropriate treatment will be provided even after the clinical trial is completed. The team has joined the insurance compensation scheme for this purpose.

\section{Outcomes $\{12\}$}

SPIRIT guidance: Primary, secondary, and other outcomes, including the specific measurement variable (eg, systolic blood pressure), analysis metric (eg, change from baseline, final value, time to event), method of aggregation (eg, median, proportion), and time point for each outcome. Explanation of the clinical relevance of chosen efficacy and harm outcomes is strongly recommended.

Primary and secondary outcomes will be measured according to a predefined timeline (Table 1). All measurements for outcome variables will be calculated as changes; values at 5, 7, 9, and 13 weeks values at 1 week ( baseline value).

\section{Primary outcomes}

The primary outcomes of this study are 1) median change ( $\mathrm{kg}$ ) in LBM, which is defined as the average of the change $(\mathrm{kg})$ from baseline and at weeks $5,7,9$, and 13 measured by DEXA, and 2) median change (kg) in handgrip strength, which is defined as the average of the change $(\mathrm{kg})$ from baseline and at weeks 5,7 , 9 , and 13 measured by a dynamometer.

\section{Secondary outcomes}

The secondary outcomes are as follows:

1) Changes $(\mathrm{kg})$ in total body mass, LBM of trunk, LBM of both upper and lower extremities, fat mass from baseline, and at week 13 from DEXA

2) Changes on the anorexia-cachexia scale

The anorexia-cachexia scale will be assessed using the Functional Assessment of Anorexia/Cachexia Treatment (FAACT) version 4, where higher the score, the better is the outcome with a range of 0-156 (15).

3) Changes in quality of life

The quality of life will be assessed using the European Organisation for Research and Treatment of Cancer Quality of Life Questionnaire (EORTC QLQ)-C30 version 3 (16). Questions 1 to 28 use a 4-point scale. The scale scores from 1 to 4: 1 ("Not at all”), 2 ("A little"), 3 ("Quite a bit"), and 4 ("Very much"). Half points are not allowed. The range is 3 . For the raw score, fewer points were considered to have a better outcome. The subsequent questions 29 and 30 use a 7-point scale. The scale scores from 1 to 7: 1 ("Very 
poor") to 7 ("Excellent"). Half points are not allowed. The range is 6 . The raw score needs to be calculated using mean values. Subsequently, a linear transformation is performed to be comparable. More points were considered to have better outcomes. Finally, the rate of toxicity with clinical significance and possible relationship to either study intervention will be obtained based on CTCAE v5.0.

4) Changes in spleen Qi deficiency questionnaire

The spleen Qi deficiency questionnaire is a validated questionnaire that includes 11 questionnaires $(17,18)$. Each item is measured on a 5 -point Likert scale, which is as follows: 0 indicates not at all or not applicable; 1 indicates a little applicable; 2 indicates moderately applicable; 3 indicates quite applicable; and 4 indicates extremely applicable. The cut-off value is 43.18 (spleen qi deficiency $>43.18$ and nonspleen qi deficiency $(\leq 43.18)$.

\section{Table 1. Timeline for outcome measurements}

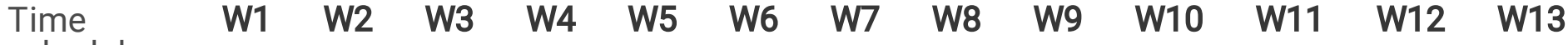
schedule

\section{Primary}

$\begin{array}{llllll}\begin{array}{l}\text { Total } \\ \text { LBM }^{1}\end{array} & \checkmark & \checkmark & \checkmark & \checkmark & \checkmark \\ \begin{array}{l}\text { Handgrip } \\ \text { strength }\end{array} & \checkmark & \checkmark & \checkmark & \checkmark & \checkmark\end{array}$

\section{Secondary}

\section{Anorexia-}

cachexia

scale

EORTC $\checkmark$

QLQ C-30²

$$
\text { SQDQ }^{3}
$$

$\checkmark$

$\checkmark$

$\checkmark$

$\checkmark$

1 Total lean body mass (LBM) and other secondary variables (total body mass, LBM of the trunk, LBM of both upper and lower extremities, and fat mass) will be measured using dual-energy X-ray absorptiometry.

${ }^{2}$ European Organisation for Research and Treatment of Cancer quality of life questionnaire C-30

${ }^{3}$ Spleen Qi deficiency pattern questionnaire

\section{Participant timeline $\{13\}$}

SPIRIT guidance: Time schedule of enrolment, interventions (including any run-ins and washouts), assessments, and visits for participants. A schematic diagram is highly recommended (see figure at 
http://www.spirit-statement.org/publications-downloads/).

\section{Sample size $\{14\}$}

SPIRIT guidance: Estimated number of participants needed to achieve study objectives and how it was determined, including clinical and statistical assumptions supporting any sample size calculations.

A sample size of 100 subjects was calculated for comparison of handgrip strength between the MIC and CPC arms with the following conditions: two-sided type I error at 0.5 , power of $90 \%$, and $1: 1$ allocation of MIC versus $C P C$ arm. Only the standard deviation (SD) of handgrip strength was used because the SD of handgrip strength was larger than that of LBM. A total sample size of 112 subjects was calculated after considering a $10 \%$ dropout rate.

\section{Recruitment $\{15\}$}

SPIRIT guidance: Strategies for achieving adequate participant enrolment to reach target sample size.

Physicians from the Department of Surgery or Medical Oncology are designated as co-investigators to screen all potentially eligible patients, even if they visit our institute for the first time.

\section{Assignment of interventions: allocation}

\section{Sequence generation $\{16 a\}$}

SPIRIT guidance: Method of generating the allocation sequence (eg, computer-generated random numbers), and list of any factors for stratification. To reduce predictability of a random sequence, details of any planned restriction (eg, blocking) should be provided in a separate document that is unavailable to those who enrol participants or assign interventions.

\section{Concealment mechanism $\{16 \mathrm{~b}\}$}

SPIRIT guidance: Mechanism of implementing the allocation sequence (eg, central telephone; sequentially numbered, opaque, sealed envelopes), describing any steps to conceal the sequence until interventions are assigned.

The independent statistician will not be allowed to contact the subjects, and this statistician will generate a randomization list using SAS, version 9.4 (Cary, NC). The randomization list will be prepared using a simple randomization procedure in a pre-determined computer with restricted access using a secure password. Eligible subjects will be randomly assigned by an independent statistician.

\section{Implementation \{16c\}}

SPIRIT guidance: Who will generate the allocation sequence, who will enrol participants, and who will assign participants to interventions 
If a patient is determined to enroll by a medical oncologist according to the protocol, the information (screening number, alphabetical initials) for this subject will be provided to the independent statistician. This statistician will assign the enrolled patient to one of the two arms (MIC or CPC) based on the predetermined randomization list. The results of the assignment will be provided to investigators and clinical staff using e-mail, just before the start of week 1.

\section{Assignment of interventions: Blinding}

\section{Who will be blinded $\{17 \mathrm{a}\}$}

SPIRIT guidance: Who will be blinded after assignment to interventions (eg, trial participants, care providers, outcome assessors, data analysts), and how.

This is an open-label study. Blinding is not possible because of the nature of each intervention. However, the person in charge of computed sequencing randomization is independent of the study team and will not know who the participants assigned to each group are.

\section{Procedure for unblinding if needed $\{17 \mathrm{~b}\}$}

SPIRIT guidance: If blinded, circumstances under which unblinding is permissible, and procedure for revealing a participant's allocated intervention during the trial.

This study is not blinded to either the participants or investigators.

\section{Data collection and management}

\section{Plans for assessment and collection of outcomes $\{18 \mathrm{a}\}$}

SPIRIT guidance: Plans for assessment and collection of outcome, baseline, and other trial data, including any related processes to promote data quality (eg, duplicate measurements, training of assessors) and a description of study instruments (eg, questionnaires, laboratory tests) along with their reliability and validity, if known. Reference to where data collection forms can be found, if not in the protocol.

Primary and secondary outcomes will be collected according to a predefined timeline (Table 1). The data for outcome variables (total LBM from DEXA, handgrip strength, survey questionnaires for the anorexiacachexia scale, EORTC quality of life), and the other data for clinical laboratory data or AEs will be primarily collected on the electronic medical reporting system of Kyung Hee University Hospital, which will be reviewed to enter into the electronic data capture tools of REDCap, hosted at Kyung Hee University. Data entry operators should be trained before data collection $(19,20)$.

\section{Plans to promote participant retention and complete follow-up $\{18 \mathrm{~b}\}$}

SPIRIT guidance: Plans to promote participant retention and complete follow-up, including list of any outcome data to be collected for participants who discontinue or deviate from intervention protocols. 
All the research teams, including the principal investigator, medical oncologist, and other staff, will have regular meetings to inspect subjects' compliance and IP accountability at each visit and withdrawal reasons for the subject. In particular, the staff who will contact subjects will communicate their discomfort related to the subject's compliance, and medical oncologists and dietitians will try to alleviate expected problems for subjects, such as abdominal discomfort and dyspepsia, which can be a hurdle to participate and complete the current study.

\section{Data management $\{19\}$}

SPIRIT guidance: Plans for data entry, coding, security, and storage, including any related processes to promote data quality (eg, double data entry; range checks for data values). Reference to where details of data management procedures can be found, if not in the protocol.

Data collection will be performed according to the protocol. These data are stored and managed in the REDCap system $(19,20)$. All data will be entered by two pre-determined staff. The quality of data will be regularly assessed by the staff and managers of the REDCap system. The developed electronic CRF in the REDCap system will automatically announce missing values and errors for values outside the predefined range. In addition, the manager will regularly export clinical data from the system to maintain data quality.

\section{Confidentiality \{27\}}

SPIRIT guidance: How personal information about potential and enrolled participants will be collected, shared, and maintained in order to protect confidentiality before, during, and after the trial.

All records that could identify the participants will be kept confidential. All documents related to this study, such as the patient's information or treatment in detail, will be recorded and classified by the subject identification code (subject study number and alphabetical initials) rather than the subject's name to protect the subject's privacy and confidentiality. Even when the results of the clinical trial are published, the identity of the participants will be kept confidential, and only the subject's study number or initials will be recorded when individual data of the subject is to be published or reported.

Clinical trial monitoring personnel and inspectors can view a subject's medical records to verify the collected information. At this time, the exposed subject's information will be handled under strict confidentiality, and the investigator will inform the subject of this fact.

Kyung Hee University Hospital will preserve and maintain the security of all data and records related to the conduct of this clinical study. The person responsible for the management and storage of the trial-related documents is the principal investigator, and access is limited to the person in charge of the study. After reporting the study results to the Institutional Review Board (IRB) of Kyung Hee University Hospital after completing the study, informed consent forms and other documents related to the study will be preserved for 3 years in accordance with the Bioethics and Safety Act in Korea. 
Plans for collection, laboratory evaluation and storage of biological specimens for genetic or molecular analysis in this trial/future use $\{33\}$

SPIRIT guidance: Plans for collection, laboratory evaluation, and storage of biological specimens for genetic or molecular analysis in the current trial and for future use in ancillary studies, if applicable.

The storage of specimens for current or future studies is not planned. All laboratory evaluations are to be performed as a blood or urine test for routine medical purposes (i.e., scheduled chemotherapy). The exceptions are plasma levels of interleukin-2, interleukin-6, and pre-albumin, which are not essential for routine clinical practice. Blood tests will be performed three times (screening, week 6 , and week 13) with 5 $\mathrm{mL}$ of whole blood each time. No genetic or molecular analyses are planned.

\section{Statistical methods}

\section{Statistical methods for primary and secondary outcomes $\{20$ a $\}$}

SPIRIT guidance: Statistical methods for analysing primary and secondary outcomes. Reference to where other details of the statistical analysis plan can be found, if not in the protocol.

All data will be analyzed using SAS (version 9.4; SAS Institute Inc., Cary, NC). Primary and secondary outcomes will be described using descriptive statistics, and the assumptions of normality will be tested for all the outcome variables to apply appropriate statistical methods: parametric (two-sample $t$-test) or non-parametric (Wilcoxon rank sum test) method. All the outcome variables will be calculated as the change or $\%$ change from the baseline measurement, [value at pre-defined schedule $(5,6,9$, and 13 weeks) - value at 1 week] or [value at pre-defined schedule $(5,6,9$, and 13 weeks) - value at 1 week]/value at 1 week. Two primary outcomes, change in handgrip strength or change in lean body mass, will be analyzed using multiple comparison procedures (Bonferroni, Holm-Bonferroni) to control the family wise error rate.

\section{Interim analyses $\{21 \mathrm{~b}\}$}

SPIRIT guidance: Description of any interim analyses and stopping guidelines, including who will have access to these interim results and make the final decision to terminate the trial.

Interim analysis is not planned for this study.

\section{Methods for additional analyses (e.g. subgroup analyses) \{20b\}}

SPIRIT guidance: Methods for any additional analyses (eg, subgroup and adjusted analyses).

Primary and secondary outcome values will be analyzed using an analysis of covariance (ANCOVA) model, adjusting for each measurement baseline as a covariate. In addition, subgroup analysis will be performed according to categorical variables such as sex or body mass index. In addition, the changes or $\%$ changes for the primary or secondary outcomes collected within each time point will be compared using a paired $t$-test or Wilcoxon signed-rank test between the MIC and CPC groups. 
Methods in analysis to handle protocol non-adherence and any statistical methods to handle missing data $\{20 \mathrm{c}\}$

SPIRIT guidance: Definition of analysis population relating to protocol non-adherence (eg, as randomised analysis), and any statistical methods to handle missing data (eg, multiple imputation).

Data for protocol non-adherence will be dealt with according to the statistical concept of intention-to-treat principle, which will prevent or minimize bias in the statistical analysis. Missing data will be handled based on the International Conference on Harmonization guidelines (ICH E9), entitled Statistical Principles for Clinical Trials. We will analyze the mechanism for the missing data to be handled with suitable methods (e.g., single imputation or multiple imputation, etc.) (21).

\section{Plans to give access to the full protocol, participant level-data and statistical code $\{31 \mathrm{c}\}$}

SPIRIT guidance: Plans, if any, for granting public access to the full protocol, participant-level dataset, and statistical code.

The full protocol will be available upon request to investigators.

\section{Oversight and monitoring}

\section{Composition of the coordinating centre and trial steering committee $\{5 \mathrm{~d}\}$}

Trials guidance: Provide information on the composition, roles and responsibilities of the coordinating centre and trial steering committee and all groups providing day to day support for the trial. There will always be a group running the trial day-to-day and providing organisational support and knowing how often they will meet, plus information on other committees providing oversight such as a Trial Steering Committee, and how often they will meet over the course of the trial, is what we need for item $5 \mathrm{~d}$. We do not need names of staff.

SPIRIT guidance: Composition, roles, and responsibilities of the coordinating centre, steering committee, endpoint adjudication committee, data management team, and other individuals or groups overseeing the trial, if applicable (see Item 21a for data monitoring committee).

Not applicable

\section{Composition of the data monitoring committee, its role and reporting structure $\{21 \mathrm{a}\}$}

SPIRIT guidance: Composition of data monitoring committee (DMC); summary of its role and reporting structure; statement of whether it is independent from the sponsor and competing interests; and reference to where further details about its charter can be found, if not in the protocol. Alternatively, an explanation of why a DMC is not needed. 
Based on the standard operating procedure of the IRB of Kyung Hee University Hospital, researchers are required to operate the data monitoring committee if the risk category is 3 (moderate risk without benefit) or higher according to the risk/benefit ratio assessment during the initial review of this study protocol. As this protocol was judged to be category 2 (more than minimal risk with possible benefit), a data monitoring committee was not mandatory.

\section{Adverse event reporting and harms $\{22\}$}

SPIRIT guidance: Plans for collecting, assessing, reporting, and managing solicited and spontaneously reported adverse events and other unintended effects of trial interventions or trial conduct.

Safety profiles including AEs will be checked and collected at every visit for chemotherapy. All AEs are standardized according to the CTCAE v5.0. In the case of dropout participants, safety profiles before dropout should be included in the safety analysis. The causal relationship between adverse events and interventions is determined in accordance with the World Health Organization-Uppsala Monitoring Centre (WHO-UMC) causality assessment $(22,23)$.

\section{Frequency and plans for auditing trial conduct $\{23\}$}

SPIRIT guidance: Frequency and procedures for auditing trial conduct, if any, and whether the process will be independent from investigators and the sponsor.

The Kyung Hee University Hospital's Human Research Protection Program (HRPP) system is comprised of the institutional leadership, HRPP manager, audit team, research compliance officer, quality improvement team, institutional review board, office for human research protection, and IRB steering committee (committee of conflict of interest). The IRB works with the HRPP to conduct a study site-visit audit and monitoring. Routine audits are conducted annually between March and November every year. Before conducting the audit, the auditor should carefully review the written plan for the audit target, consent form, and documents submitted to the IRB, and establish detailed audit plans. The auditor assigns an audit number to each audit according to the order of execution of the audit. The audit number shall be prepared as "Year-Audit Type + Audit Order." At an audit initiation meeting, an auditor explains the purpose, scope, and procedure of the audit to the investigator and receives research-related documents. Based on the predefined "Study Site Visit Checklist for Kyung Hee University," the auditor shall inspect the data that may affect the reliability and integrity of the trial, such as documents related to consent of the participants (such as consent form), suitability of the target (selected/exclusion criterion), records related to administration/application of medicines/medical devices, and items to be evaluated for primary validity/safety of the research. The auditor may secure a copy of the evidence, if necessary. A closing meeting of the audit shall be attended by an investigator in charge of the research, including a principal investigator, and an auditor who conducted the related audit of the research. The auditor shall conduct an interview with the person in charge of the relevant task at the closing meeting of the audit to check whether the findings are true. In addition, major findings and suggestions are explained verbally, and the audit results should be discussed. The auditor may reflect modifications in the audit results based on the 
details discussed with the investigator. If there are any lost, incomplete, or incorrect data, an investigator may submit the data to an auditor within 3 days of the audit. The auditor may check the submitted data and reflect the results of the discussion in the audit results. The auditor shall record the list of documents reviewed during the audit and the discussion with the investigators in the "Study Site Visit Checklist for Kyung Hee University." Of note, all the processes are completely independent of the investigators and the sponsor of this study. The IRB and HRPP of Kyung Hee University Hospital are accredited by the Association for the Accreditation of Human Research Protection Programs, Inc. (AAHRPP).

\section{Plans for communicating important protocol amendments to relevant parties (e.g. trial participants, ethical committees) $\{25\}$}

SPIRIT guidance: Plans for communicating important protocol modifications (eg, changes to eligibility criteria, outcomes, analyses) to relevant parties (eg, investigators, REC/IRBs, trial participants, trial registries, journals, regulators).

In the event of alterations, the amended study protocol should be submitted to the IRB for review. A report of amendments made to the previously approved study protocol is requested for submission. In the report, the following items are to be included: 1) description and explanation of amendments made, 2) reason for making amendments, 3) any AEs that occurred under the original research protocol, and 4) expected AEs owing to the amendments. Because this clinical trial is conducted after obtaining approval from the Ministry of Food and Drug Safety of Korea, the amended study protocol and related documents are necessary to confirm whether the Minister of Food and Drug Safety approved the change. However, changes that do not significantly affect the safety of the subjects or the reliability of the test results can be waived.

\section{Dissemination plans $\{31$ a $\}$}

SPIRIT guidance: Plans for investigators and sponsor to communicate trial results to participants, healthcare professionals, the public, and other relevant groups (eg, via publication, reporting in results databases, or other data sharing arrangements), including any publication restrictions.

The study results will be published in a peer-reviewed journal. Before that, the abstract of the results can be presented at an academic conference.

\section{Discussion}

Trials guidance: This should include a discussion of any practical or operational issues involved in performing the study and any issues not covered in other sections.

When the investigators planned this clinical trial for the first time, it was planned that the MIC group included a total of seven kinds of drug and non-drug interventions, whereas the CPC group included only one routine nutritional counseling. Because the researchers thought that to clearly observe the effect of multimodal experimental interventions, it was necessary to remove other treatments that could potentially 
cause therapeutic effects in the control group, an issue related to clinical equipoise between the experimental and control groups was raised in the initial IRB deliberation. However, in response to the opinion of the IRB committee, the study team decided to include MA and apply the usual care in real-world practice to the control group. It was decided that MA would be provided when predefined criteria were met for participants allocated to the CPC group. In addition, nutritional counseling will be provided one more time at the end of the study (week 13) to increase the patient's dietary sand nutritional status.

At the time of writing this manuscript, it has been reported that some participants who have already experienced interventions in the MIC group sometimes complained of abdominal bloating from taking ONS. One pack of ONS has a volume of $200 \mathrm{~mL}$ and is to be taken twice a day. In addition to the issues of IP, weekly visits to the hospital for exercise treatment were also difficult in some cases. If there are too many missing parts of the planned interventions, there is a risk of bias in the interpretation of the results after the study is completed. Although this study is ongoing and compliance for IP has not yet been fully investigated, there might be a concern that a number of concomitant interventions may be difficult for patients to implement as intended by a physician in real-world practice. Nevertheless, if multimodal interventions are found to be effective in improving cachexia in this study, it may be possible to provide valuable knowledge to help patients.

\section{Trial status}

Trials guidance: Authors should report the protocol version number and date, the date recruitment began, and the approximate date when recruitment will be completed.

The registration date for the first participant was September 15, 2020. At the time of submission, 24 of the 112 subjects were enrolled. The estimated study period is 18 months. The most recent protocol is version 2.5, revised as of February 22, 2021.

\section{Abbreviations}

Trials guidance: If abbreviations are used in the text they should be defined in the text at first use, and a list of abbreviations should be provided.

CC: cancer cachexia

MA: megestrol acetate

FDA: United States of America Food and Drug Administration

EMA: European Medicines Agency

NSAID: Non-steroidal anti-inflammatory drugs

O3FA: omega-3-fatty acid 
ONS: oral nutritional supplement

CAM: complementary and alternative medicine

MIC: multi-modal intervention care

CPC: conventional Palliative Care

ECOG: Eastern Cooperative Oncology Group

DEXA: dual-energy X-ray absorptiometry

IP: investigational product

CTCAE: Common Terminology Criteria for Adverse Events

LBM: total lean body mass

FAACT: Functional Assessment of Anorexia/Cachexia Treatment

EORTC QLQ: European Organisation for Research and Treatment of Cancer quality of life questionnaire IRB: the institutional review board

AE: adverse event

WHO-UMC : World Health Organization-Uppsala Monitoring Centre

HRPP: Human Research Protection Program

AAHRPP: Association for the Accreditation of Human Research Protection Programs, Inc.

\section{Declarations}

Trials guidance: All manuscripts must contain the following subheadings:

- Acknowledgements

- Authors' contributions

- Funding

- Availability of data and material

- Ethics approval and consent to participate

- Consent for publication

- Competing interests

- Authors' information (optional) 


\section{Acknowledgements}

Trials guidance: Please acknowledge anyone who contributed towards the article who does not meet the criteria for authorship including anyone who provided professional writing services or materials. Authors should obtain permission to acknowledge from all those mentioned in the Acknowledgements section. See our editorial policies for a full explanation of acknowledgements and authorship criteria. If you do not have anyone to acknowledge, please write "Not applicable" in this section.

Group authorship (for manuscripts involving a collaboration group): if you would like the names of the individual members of a collaboration Group to be searchable through their individual PubMed records, please ensure that the title of the collaboration Group is included on the title page and in the submission system and also include collaborating author names as the last paragraph of the "Acknowledgements" section. Please add authors in the format First Name, Middle initial(s) (optional), Last Name. You can add institution or country information for each author if you wish, but this should be consistent across all authors. Please note that individual names may not be present in the PubMed record at the time a published article is initially included in PubMed as it takes PubMed additional time to code this information.

Not applicable.

\section{Authors' contributions $\{31 \mathrm{~b}\}$}

SPIRIT guidance: [31b] - Authorship eligibility guidelines and any intended use of professional writers.

Trials guidance: The individual contributions of authors to the manuscript should be specified in this section. Guidance and criteria for authorship can be found in our editorial policies. Please use initials to refer to each author's contribution in this section, for example: "AB is the Chief Investigator; she conceived the study, led the proposal and protocol development. CD contributed to study design and to development of the proposal. EF was the lead trial methodologist. All authors read and approved the final manuscript.

$\mathrm{KYL}$ is the Chief Investigator and has been awarded a grant for this study. CHM and BHK conceived the study and led proposal and protocol development. CHM is primarily responsible for subject registration. $\mathrm{JC}$ contributed to the exercise of the protocol. WSK developed the psychiatric part of the intervention. Nutritional counseling and ONS were developed and supported by KK and MW. The CAM part of this study was developed by JL. IKH guided diagnostic methods, including DEXA. BHK was the lead trial methodologist and constructed an electronic case report form. All authors have read and approved the final manuscript.

\section{Funding \{4\}}

SPIRIT guidance: Sources and types of financial, material, and other support. 
Trials guidance: All sources of funding for the research reported should be declared. You will be required to include a copy of the original funding document and an English translation of this document as an additional file on submission, which will be checked against this declaration. The role of the funding body in the design of the study and collection, analysis, and interpretation of data and in writing the manuscript should be declared.

This research is supported by the Basic Science Research Program through the National Research Foundation of Korea (NRF) funded by the Ministry of Science, ICT \& Future Planning (2017M3A9E4065330). However, the funding body is independent of the study team or study details and is not involved in the design of the study, data collection, analysis and interpretation, and drafting of the manuscript.

\section{Availability of data and materials $\{29\}$}

SPIRIT guidance: Statement of who will have access to the final trial dataset, and disclosure of contractual agreements that limit such access for investigators.

Trials guidance: Please do not include any baseline or pilot data in your study protocol. The Editorial Office will ask you to remove this if it is included. Please declare here who will have access to the final trial dataset and disclose contractual agreements that limit such access for investigators.

This study is an investigator-initiated trial, and the study team includes the principal investigator and subinvestigators who can access the study data. Because the grant body is not directly involved in the study design, conduct of the study, and data analysis, investigators do not need to contract with others to gain access to data.

\section{Ethics approval and consent to participate $\{24\}$}

SPIRIT guidance: Plans for seeking research ethics committee/institutional review board (REC/IRB) approval.

Trials guidance: Trials do not consider study protocols for studies without ethical approval. You will be required to provide a copy of the original ethical approval document and an English translation of this document as an additional file on submission, which will be checked against this declaration. The name of the ethics committee that approved the study and the committee's reference number (if applicable) should be declared. Details of authors' intentions to obtain consent to participate in the study from participants (or their parent or legal guardian in the case of children under 16) should be declared. "eg. $A B C$ Ethical Review Board ABC123456. Written, informed consent to participate will be obtained from all participants"

In Korea, according to the Pharmaceutical Affairs Act, clinical trials are required to obtain approval from the Ministry of Food and Drug Safety and the IRB of the institution where the clinical trial is being 
conducted. The Kyung Hee University Hospital IRB approval number is KHUH 2019-08-084-008. This study is also approved by the Ministry of Food and Drug Safety.

\section{Consent for publication $\{32\}$}

SPIRIT guidance: Model consent form and other related documentation given to participants and authorised surrogates.

Trials guidance: Please do not include any baseline or pilot data in your study protocol. The Editorial Office will ask you to remove this if it is included. If you have included any details, images or videos relating to an individual person, written informed consent for the publication of these details must be obtained from that person (or their parent or legal guardian in the case of children under 18) and declared in this section. Please also state whether you will be willing to provide a model consent form on request. If this section does not apply, please state "Not applicable".

A model consent form can be provided upon request. However, the consent form was written in Korean.

\section{Competing interests $\{28\}$}

SPIRIT guidance: Financial and other competing interests for principal investigators for the overall trial and each study site.

Trials guidance: All financial and non-financial competing interests must be declared in this section. See our editorial policies for a full explanation of competing interests. If you are unsure whether you or any of your co-authors have a competing interest please contact the editorial office. Please use the authors initials to refer to each authors' competing interests in this section. If you do not have any competing interests, please state: "The authors declare that they have no competing interests" in this section.

The authors declare that they have no competing interests.

\section{Authors' information (optional)}

Trials guidance: This section is optional.

You may choose to use this section to include any relevant information about the author(s) that may aid the reader's interpretation of the article, and understand the standpoint of the author(s). This may include details about the authors' qualifications, current positions they hold at institutions or societies, or any other relevant background information. Please refer to authors using their initials. Note this section should not be used to describe any competing interests.

\section{References}

Trials guidance: Examples of the Vancouver reference style are shown below. 
See our editorial policies for author guidance on good citation practice

Web links and URLs: All web links and URLs, including links to the authors' own websites, should be given a reference number and included in the reference list rather than within the text of the manuscript. They should be provided in full, including both the title of the site and the URL, as well as the date the site was accessed, in the following format: The Mouse Tumor Biology Database.

http://tumor.informatics.jax.org/mtbwi/index.do. Accessed 20 May 2013. If an author or group of authors can clearly be associated with a web link, such as for weblogs, then they should be included in the reference.

\section{Example reference style:}

Article within a journal

Smith JJ. The world of science. Am J Sci. 1999;36:234-5.

Article within a journal (no page numbers)

Rohrmann S, Overvad K, Bueno-de-Mesquita HB, Jakobsen MU, Egeberg R, Tjønneland A, et al. Meat consumption and mortality - results from the European Prospective Investigation into Cancer and Nutrition. BMC Medicine. 2013;11:63.

Article within a journal by DOI

Slifka MK, Whitton JL. Clinical implications of dysregulated cytokine production. Dig J Mol Med. 2000; doi:10.1007/s801090000086.

Article within a journal supplement

Frumin AM, Nussbaum J, Esposito M. Functional asplenia: demonstration of splenic activity by bone marrow scan. Blood 1979;59 Suppl 1:26-32.

Book chapter, or an article within a book

Wyllie AH, Kerr JFR, Currie AR. Cell death: the significance of apoptosis. In: Bourne GH, Danielli JF, Jeon KW, editors. International review of cytology. London: Academic; 1980. p. 251-306.

OnlineFirst chapter in a series (without a volume designation but with a DOI)

Saito $\mathrm{Y}$, Hyuga $\mathrm{H}$. Rate equation approaches to amplification of enantiomeric excess and chiral symmetry breaking. Top Curr Chem. 2007. doi:10.1007/128_2006_108.

Complete book, authored 
Blenkinsopp A, Paxton P. Symptoms in the pharmacy: a guide to the management of common illness. 3rd ed. Oxford: Blackwell Science; 1998.

\section{Online document}

Doe J. Title of subordinate document. In: The dictionary of substances and their effects. Royal Society of Chemistry. 1999. http://www.rsc.org/dose/title of subordinate document. Accessed 15 Jan 1999.

\section{Online database}

Healthwise Knowledgebase. US Pharmacopeia, Rockville. 1998. http://www.healthwise.org. Accessed 21 Sept 1998.

\section{Supplementary material/private homepage}

Doe J. Title of supplementary material. 2000. http://www.privatehomepage.com. Accessed 22 Feb 2000.

University site

Doe, J: Title of preprint. http://www.uni-heidelberg.de/mydata.html (1999). Accessed 25 Dec 1999.

FTP site

Doe, J: Trivial HTTP, RFC2169. ftp://ftp.isi.edu/in-notes/rfc2169.txt (1999). Accessed 12 Nov 1999.

\section{Organization site}

ISSN International Centre: The ISSN register. http://www.issn.org (2006). Accessed 20 Feb 2007.

\section{Dataset with persistent identifier}

Zheng L-Y, Guo X-S, He B, Sun L-J, Peng Y, Dong S-S, et al. Genome data from sweet and grain sorghum (Sorghum bicolor). GigaScience Database. 2011. http://dx.doi.org/10.5524/100012.

1. Fearon K, Strasser F, Anker SD, Bosaeus I, Bruera E, Fainsinger RL, et al. Definition and classification of cancer cachexia: an international consensus. The lancet oncology. 2011;12(5):489-95.

2. von Haehling S, Anker SD. Cachexia as a major underestimated and unmet medical need: facts and numbers. Journal of cachexia, sarcopenia and muscle. 2010;1(1):1-5.

3. Temel JS, Abernethy AP, Currow DC, Friend J, Duus EM, Yan Y, et al. Anamorelin in patients with nonsmall-cell lung cancer and cachexia (ROMANA 1 and ROMANA 2): results from two randomised, double-blind, phase 3 trials. The lancet oncology. 2016;17(4):519-31.

4. Dewys WD, Begg C, Lavin PT, Band PR, Bennett JM, Bertino JR, et al. Prognostic effect of weight loss prior to chemotherapy in cancer patients. Eastern Cooperative Oncology Group. Am J Med. 1980;69(4):491-7. 
5. Lim S, Brown JL, Washington TA, Greene NP. Development and progression of cancer cachexia: Perspectives from bench to bedside. Sports Medicine and Health Science. 2020;2(4):177-85.

6. Argilés JM, Busquets S, Stemmler B, López-Soriano FJ. Cancer cachexia: understanding the molecular basis. Nature reviews Cancer. 2014;14(11):754-62.

7. Suzuki H, Asakawa A, Amitani H, Nakamura N, Inui A. Cancer cachexia-pathophysiology and management. Journal of gastroenterology. 2013;48(5):574-94.

8. Arends J, Strasser F, Gonella S, Solheim TS, Madeddu C, Ravasco P, et al. Cancer cachexia in adult patients: ESMO Clinical Practice Guidelines $\$. ESMO Open. 2021;6(3):100092.

9. Ruiz Garcia V, López-Briz E, Carbonell Sanchis R, Gonzalvez Perales JL, Bort-Martí S. Megestrol acetate for treatment of anorexia-cachexia syndrome. Cochrane Database of Systematic Reviews. 2013(3).

10. Loprinzi CL, Schaid DJ, Dose AM, Burnham NL, Jensen MD. Body-composition changes in patients who gain weight while receiving megestrol acetate. Journal of clinical oncology : official journal of the American Society of Clinical Oncology. 1993;11(1):152-4.

11. Dev R, Del Fabbro E, Bruera E. Association between megestrol acetate treatment and symptomatic adrenal insufficiency with hypogonadism in male patients with cancer. Cancer. 2007;110(6):1173-7.

12. Solheim TS, Fearon KC, Blum D, Kaasa S. Non-steroidal anti-inflammatory treatment in cancer cachexia: a systematic literature review. Acta oncologica (Stockholm, Sweden). 2013;52(1):6-17.

13. Aapro M, Arends J, Bozzetti F, Fearon K, Grunberg SM, Herrstedt J, et al. Early recognition of malnutrition and cachexia in the cancer patient: a position paper of a European School of Oncology Task Force. Annals of oncology : official journal of the European Society for Medical Oncology / ESMO. 2014;25(8):1492-9.

14. Fearon K, Strasser F, Anker SD, Bosaeus I, Bruera E, Fainsinger RL, et al. Definition and classification of cancer cachexia: an international consensus. The lancet oncology. 2011;12(5):489-95.

15. Ribaudo JM, Cella D, Hahn EA, Lloyd SR, Tchekmedyian NS, Von Roenn J, et al. Re-validation and shortening of the Functional Assessment of Anorexia/Cachexia Therapy (FAACT) questionnaire. Qual Life Res. 2000;9(10):1137-46.

16. Fayers P, Bottomley A. Quality of life research within the EORTC-the EORTC QLQ-C30. European Organisation for Research and Treatment of Cancer. European journal of cancer (Oxford, England : 1990). 2002;38 Suppl 4:S125-33.

17. Kim J, Kim H, Kim KH. Effects of Bu-Zhong-Yi-Qi-Tang for the treatment of functional dyspepsia: a feasibility study protocol. Integrative Medicine Research. 2017;6(3):317-24.

18. Kim J, Park J-W, Ko S-J, Jeon S-H, Kim J-W, Yeo I, et al. Effects of a Herbal Medicine, Yukgunja-Tang, on Functional Dyspepsia Patients Classified by 3-Dimensional Facial Measurement: A Study Protocol for Placebo-Controlled, Double-Blind, Randomized Trial. Evidence-Based Complementary and Alternative Medicine. 2017;2017:2894507.

19. Harris PA, Taylor R, Thielke R, Payne J, Gonzalez N, Conde JG. Research electronic data capture (REDCap)-a metadata-driven methodology and workflow process for providing translational research 
informatics support. J Biomed Inform. 2009;42(2):377-81.

20. Harris PA, Taylor R, Minor BL, Elliott V, Fernandez M, O'Neal L, et al. The REDCap consortium: Building an international community of software platform partners. J Biomed Inform. 2019;95:103208.

21. RUBIN DB. Inference and missing data. Biometrika. 1976;63(3):581-92.

22. Edwards IR, Aronson JK. Adverse drug reactions: definitions, diagnosis, and management. Lancet. 2000;356(9237):1255-9.

23. Nebeker JR, Barach P, Samore MH. Clarifying adverse drug events: a clinician's guide to terminology, documentation, and reporting. Annals of internal medicine. 2004;140(10):795-801.

\section{Figures}

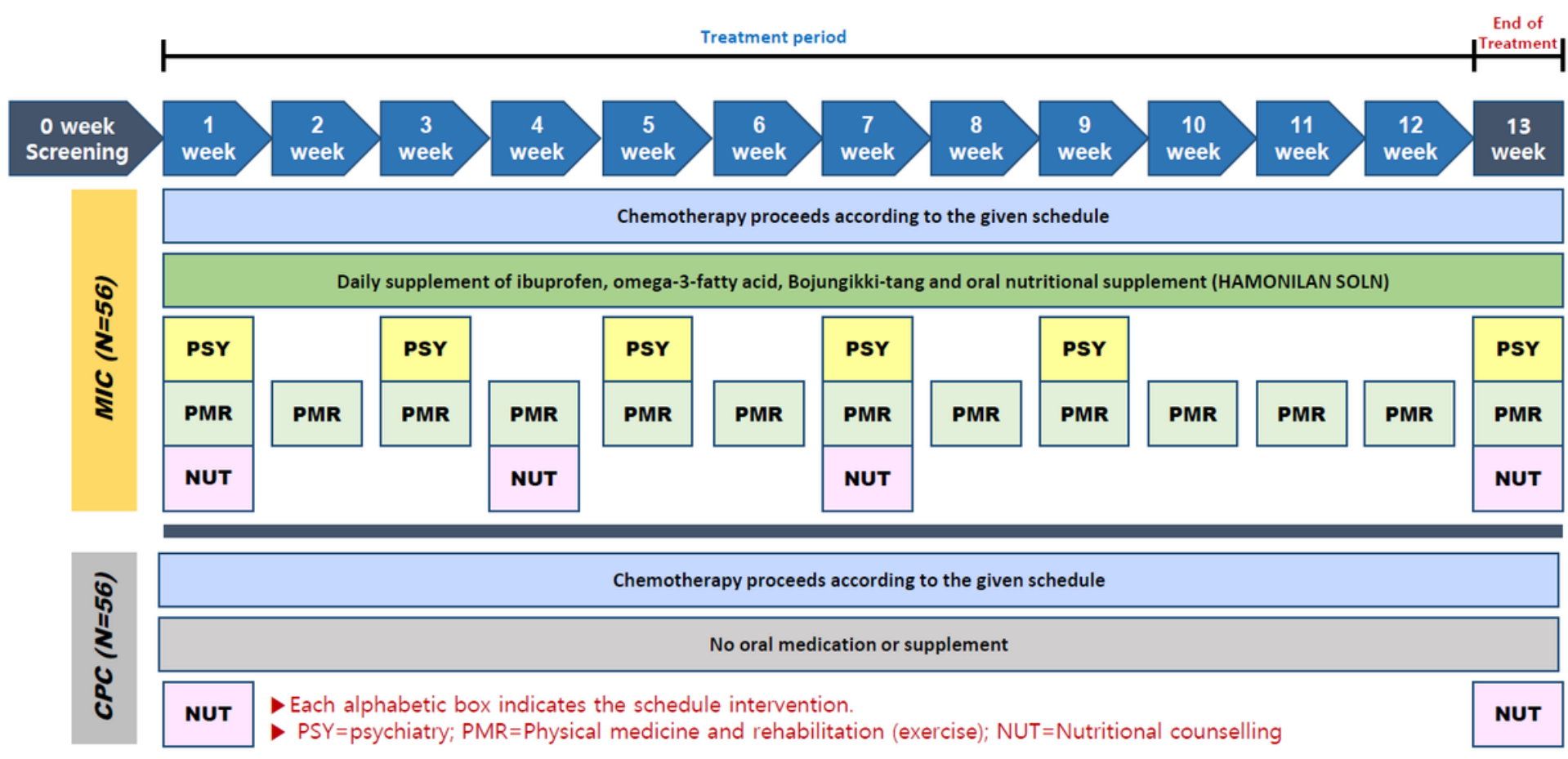

\section{Figure 1}

Time schedule of enrolment, interventions (including any run-ins and washouts), assessments, and visits for participants. A schematic diagram is highly recommended 